\title{
The USNA MIDN Microdosimeter Instrument
}

\author{
V. L. Pisacane ${ }^{1}$, J. F. Ziegler ${ }^{2}$, M. E. Nelson ${ }^{3}$, Q. Dolecek ${ }^{4}$, J. Heyne ${ }^{5}$, T. Veade ${ }^{6}$, \\ United States Naval Academy, Annapolis, MD, 21402 USA \\ A.B. Rosenfeld ${ }^{7}$ \\ University of Wollongong, NSW, 2522 Australia \\ F.A. Cucinotta ${ }^{8}$ \\ NASA Lyndon B. Johnson Space Center, Houston, TX, 77058 USA \\ M. Zaider ${ }^{9}$ \\ Memorial Sloan-Kettering Cancer Center, New York, NY, 10021 USA
}

\author{
J.F. Dicello ${ }^{10}$ \\ The Johns Hopkins University School of Medicine, Baltimore, MD, 21231 USA
}

This paper describes the MIcroDosimetry iNstrument (MIDN) mission now under development at the United States Naval Academy. The instrument is manifested to fly on the MidSTAR-1 spacecraft, which is the second spacecraft to be developed and launched by the Academy's faculty and midshipmen. Launch is scheduled for 1 September 2006 on an ATLAS-5 launch vehicle. MIDN is a rugged, portable, low power, low mass, solid-state microdosimeter designed to measure in real time the energy distributions of energy deposited by radiation in microscopic volumes. The MIDN microdosimeter sensor is a reverse-biased silicon p-n junction array in a Silicon-On-Insulator (SOI) configuration. Microdosimetric frequency distributions as a function of lineal energies determine the radiation quality factors in support of radiation risk estimation to humans.

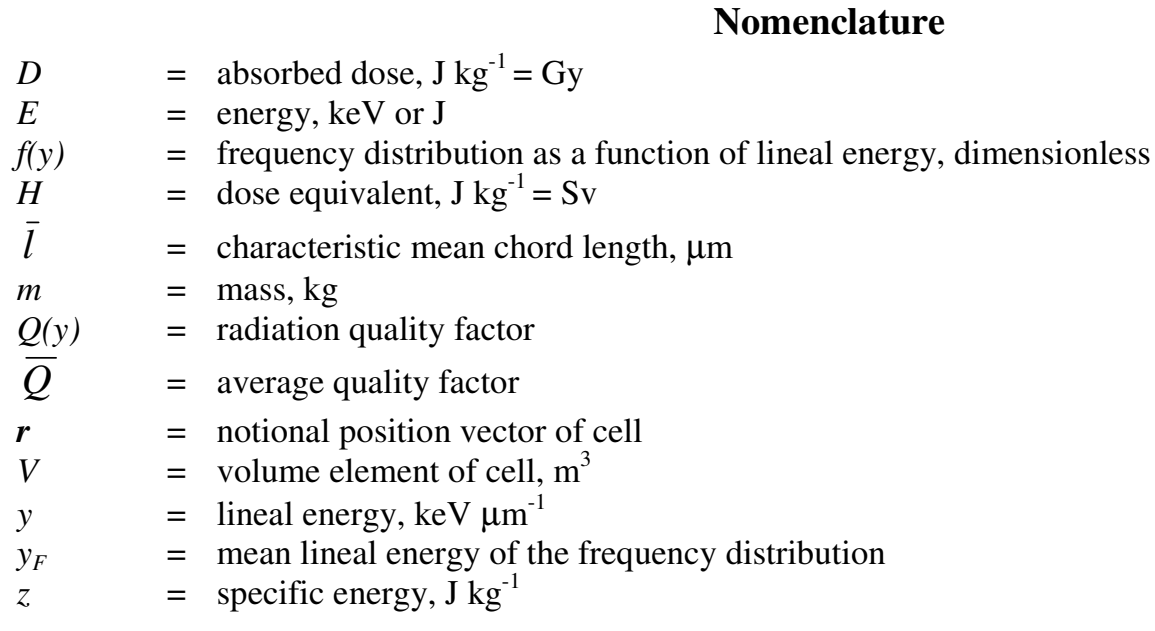

\footnotetext{
${ }_{1}^{1}$ R. A. Heinlein Professor of Aerospacel Engineering, 590 Holloway Road, Mail Stop 11B, AIAA Fellow

2 Professor of Electrical Engineering, 590 Holloway Road, Mail Stop 14B

${ }^{3}$ Professor of Mechanical Engineering, 590 Holloway Road, Mail Stop 11C

${ }^{4}$ Consultant, 590 Holloway Road, Mail Stop 11B

${ }^{5}$ Midshipman First Class, Bancroft Hall, PO Box 12506

${ }^{6}$ Midshipman First Class, Bancroft Hall, PO Box 14812

${ }^{7}$ Professor, Director Centre for Medical Radiation Physics

${ }^{8}$ Project Scientist and Radiation Health Officer, 2101 NASA Road 1

9 Attending and Head of Brachytherapy Physics, 1275 York Avenue

${ }^{10}$ Professor of Radiation Oncology and Professor of Oncology, 401 North Broadway
} 


\section{Introduction}

$\mathrm{T}$ he space radiation environment is significantly enhanced relative to that on Earth due to the reduced protection of the atmosphere and the geomagnetic field. High energy radiation composed of electrons, protons, helium, and heavier ions originate from several sources including trapped radiation, galactic cosmic rays, and solar particle events. Astronauts, therefore, generally receive a greater dose per unit time in space and there is the potential for increased likelihood of cancer and other diseases as well as life shortening. While shielding can reduce their exposure to the ambient environment, it also can produce significant secondary radiation. The exposure to radiation in space in one day can be close to the dose received on Earth in one year, excluding radon. Large solar events can significantly increase radiation exposure. The amount of time that astronauts can spend in space is limited by their lifetime dose equivalent, which is the product of the physical absorbed dose and the mean quality factor. The average quality factor represents the relative effectiveness of a radiation field compared to gamma rays. The quality factor depends on the distribution in linear energy transfer (LET) or lineal energy ( $y$ ) which in turn depend on the type and energy of the radiation as well as the geometry of sensitive cellular sites. The LET-based quality factor can be calculated (but not measured) if the probability distribution as a function of LET is known, which varies with energy and particle type. Alternatively, a microdosimetric-based quality factor can be obtained if microdosimetric spectra for the radiations and sites of interest are known. Calculational tools exist for obtaining microdosimetric distributions for radiation fields if the energy and particle fluences are known at the point of interest (typically, from particle transport calculations). However, experimental microdosimetry (essentially, using tissue equivalent gas) remains largely a research tool; therefore there is a need for rugged portable microdosimeters capable of obtaining frequency distributions in space missions of long durations.

The purpose of MIDN is to directly determine quality factors by measuring the microdosimetric spectra as a function of lineal energy in sizes comparable to those of tissue cells. The potential health risk due to radiation is one of four type 1 (most serious) potential barriers to human exploration ${ }^{1}$. Presently, there are no protective or preventive biological countermeasures that can assure the health and safety of astronauts during long-term missions. To assure safe long-duration human missions:

- The risks of short- and long-term health effects from radiation need to be better understood;

- Protective and/or preventive countermeasures need to be developed;

- Synergistic interactions between microgravity and other environmental factors and radiation need to be understood ${ }^{2,3,4}$;

- Electronics systems need to be tolerant of high radiation environments; and

- Instruments need to be developed to assess the radiation environment and its quality in real time.

There are potentially six approaches to reduce the probability of untoward radiation effects ${ }^{5}$ :

$\circ \quad$ Operations -restricting trajectories and limiting mission time;

- Shielding -at the expense of reduced payload and mission effectiveness;

- Screening -eliminating electronic parts or humans with increased susceptibility;

- Surveillance - monitoring the radiation environment to predict times of increased radiation activity;

- Prophylaxes - utilizing chemopreventive treatments: utilizing chemoprotective drugs, transfusions, bone marrow transplants, and/or gene therapy.

The recommended policy on radiation risks in low-Earth orbit is to limit exposure to less than a three percent increase in the lifetime potential for cancer mortality ${ }^{3,4,6}$.

\section{Mission Description}

The MidSTAR (Midshipmen Space Technology Applications Research) series of spacecraft, under development at the United States Naval Academy (USNA), is intended to be a low-cost, quick response platform accommodating small payloads approved by the Department of Defense Space Experiments Review Board (SERB) and awaiting launch through the Space Test Program of the Department of Defense Space and Missile Systems Command. MidSTAR satellites are Class D spacecraft, to be produced at minimum cost with a correspondingly higher technical risk resulting from compromises in design, test, production and operation. They are intentionally simple in design 
and rugged in construction, using commercial off-the-shelf "plug-and-play" components to the greatest extent possible, see Table 1 for NASA mission classifications ${ }^{7}$. MidSTAR is a project of the United States Naval Academy (USNA) Small Satellite Program (SSP) ${ }^{8,9}$.

MidSTAR-1 is the first of the MidSTAR series of spacecraft commissioned by the STP and is scheduled for launch on a Lockheed-Martin Atlas V from Cape Canaveral on the STP-1 mission on 1 September 2006. This prototype mission is to demonstrate the viability of the MidSTAR program. MidSTAR-1 will carry two STP-approved experiments as well as additional USNA, Naval Postgraduate School (NPS), and NASA experiments. The spacecraft, illustrated in Figure 1(a), has a mass of $120 \mathrm{~kg}$ and will be placed into a 492-km near circular orbit with a $46^{\circ}$ inclination. Its orbital period will be approximately 5667 seconds, and it will make approximately 15.2 revolutions per day. The spacecraft will be operated from the spacecraft ground control station at the USNA. The downlink data rate will be $68.4 \mathrm{kbps}$ at a frequency of $2.022 \mathrm{GHz}$.

\begin{tabular}{|l|l|}
\hline Class A & Minimum Risk \\
\hline Class B & Risk/Cost Compromise \\
\hline Class C & Single Purpose, Repeat Mission Possible, Some Risk Allowed \\
\hline Class D & Routine, Rapid Mission, or Proof of Concept, More Risk Allowed \\
\hline
\end{tabular}

Table 1. Mission Classification

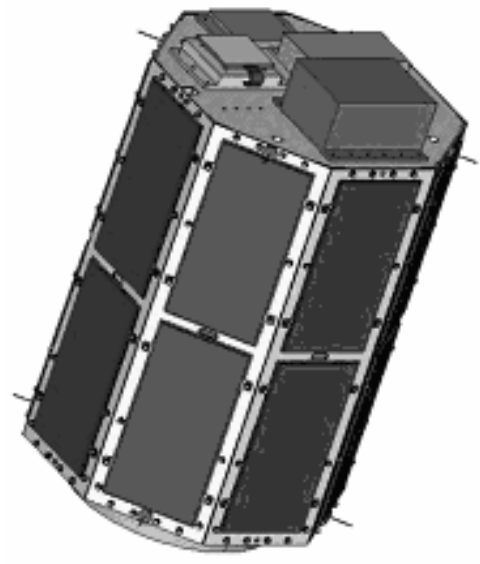

(a) MidSTAR Spacecraft

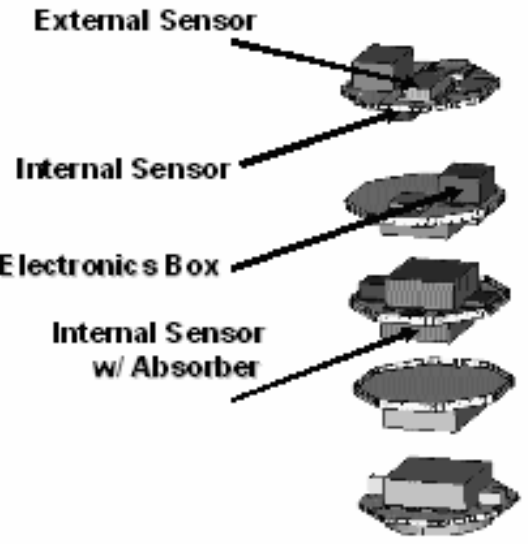

(b) MIDN Component Locations

Figure 1. MidSTAR configuration

\section{Microdosimetry}

Microdosimetry is the study of the physical processes associated with the transfer of energy from radiation to matter and their relationship to the subsequent chemical and biological consequences. ${ }^{10}$ It provides perhaps the most universally accepted techniques for determining radiation qualities and dose equivalents in microscopic size volumes and is useful for all types of radiation. It provides the physical underpinnings for the measurement of radiation risk and is vitally important to both radiation therapy and radiation protection. Microdosimetry was developed through attempts to measure the distribution of the linear energy transfer (LET), the energy lost by a particle per unit path length traversed, when radiation interacts with elemental volumes, the size of a cell or subcellular structures such as the nucleus or chromosomes. The microdosimetry energy spectrum is unique in that it can be analyzed to give separate photon and neutron dose rates, average quality factors, and in conjunction with a dose measurement, the dose equivalent rates. 
Health risk due to radiation is generally assumed to depend on the specific energy deposited by the radiation. If radiation traverses a volume characterized by mass $m$ and deposits energy $E$ the specific energy $z$ in the material is given by

$$
z=E / m
$$

The corresponding absorbed dose $D$ is then defined by

$$
D(\mathbf{r})=\lim _{V \rightarrow 0} \bar{z}
$$

where $V$ is the volume of the mass element, $\boldsymbol{r}$ denotes its location, and $\bar{z}$ is the average value of the specific energy where the limit in Equation 2 is taken at constant density. A microdosimetric quantity analogous to the LET and directly related to the specific energy is the lineal energy, $y$, where

$$
y=E / \bar{l}
$$

where $\bar{l}$ is the mean cord length of the volume element. For purposes of radiation protection the frequency distributions in the detector medium must approximate those in tissue or be converted to those in tissue. With the frequency distributions determined as a function of lineal energy $y$, the quality factor $Q(y)$ can be determined from ICPR $-60^{11}$. The human risk to radiation can then be characterized by the dose equivalent $H$ given by

$$
H=\frac{D \int Q(y) y f(y) d y}{y_{F}}=D \bar{Q}
$$

where $Q(y)$ is the quality factor, $\bar{Q}$ is the average quality factor, $f(y)$ is the frequency distribution, and $y_{F}$ is the mean lineal energy of the frequency distribution, i.e.,

$$
y_{F}=\int_{0}^{\infty} y f(y) d y
$$

The physical dose can be measured by a variety of active and/or passive techniques. Historically, radiation quality has been most often evaluated retrospectively by processing particle tracks in plastic films recovered after exposure. The track distributions are used to estimate particle frequencies as a function of linear energy transfer, LET, and the resulting quality factor. The delay in obtaining information restricts the protective measures that can be invoked. Gas proportional counters have been used to obtain real-time estimates of the radiation quality and dose equivalent by measuring the dose distribution function $f(y)$ of lineal energy. Such information can be evaluated by the astronauts and/or downloaded. However, proportional counters are delicate devices subject to changes in response from outgassing and contamination, and they are sensitive to vibrations and noise. Solid-state detectors have been also used to obtain microdosimetric spectra, but earlier devices had excessively high noise and insufficient range of energies ${ }^{10,12}$. More recently, it has been proposed that specialized integrated circuits could address the weaknesses of previous devices ${ }^{13}$. As a consequence, the authors are developing such a system for the MidSTAR program ${ }^{14}$.

The quantity measured in the solid-state microdosimeter is the liberated charge in the sensitive volume rather than energy, and this is then converted to a voltage pulse height which is proportional to the energy deposited $E$. This relationship must be determined by calibration of the microdosimeter. The characteristics of the microdosimeter volume provide the mean chord length $\bar{l}$ and mass $m$ of each cell. With $E$ and $m$ known, to the extent that volume and interface effects can be ignored, the absorbed dose $D$ in the detector material can be determined from Equations 1 and 2. The mean lineal energy $\bar{l}$ is determined geometrically. From the the lineal energy $y$ associated with an 
event, the frequency distribution $f(y)$ and quality factor $Q(y)$ are determined. Then Equation 4 can be used to calculate the dose equivalent $H$.

The density of the matter in the detector is arranged such that the energy loss in the microdosimeter volume and tissue equivalent cell is nearly the same for the same trajectories. The energy loss $E$ is proportional to the product of the density and the chord length so that

$$
\begin{aligned}
& E_{t}=E_{s} \\
& \rho_{t} l_{t}=\rho_{s} l_{s}
\end{aligned}
$$

and it follows that for the same energy deposition, the equivalent chord length in tissue relative to silicon is

$$
l_{s}=\frac{\rho_{s}}{\rho_{t}} l_{s}
$$

This permits the measurements in the silicon microdosimeter to be interpreted for equivalent tissue measurements.

\section{Instrumentation Overview}

MIDN is a rugged, portable, low power and mass, solid-state microdosimeter designed to measure in real time the energy distributions of radiation deposited in microscopic volumes. The sensor was developed at the Centre for Medical Radiation Physics of the University of Wollongong utilizing the Fujitsu diode array silicon on insulator (SOI) device ${ }^{12,13}$. The supporting space electronics and communications subsystems are under development at the USNA in collaboration with the Johns Hopkins University School of Medicine. The MidSTAR MIDN instrument consists of three separate sensor modules and single electronics module that provides power and processes the outputs of the sensors. The design goals, independent of the absorber mass, are a mass $<3 \mathrm{~kg}$ and power $<1 \mathrm{~W}$. A design with a single microdosimeter sensor module would require $<1 \mathrm{~kg}$ in mass and $<1 \mathrm{~W}$ of power. The MidSTAR mission provides a space flight opportunity to test and evaluate a preliminary version of the MIDN instrument. Because of the low power and mass, potential use of the instrument includes the International Space Station, robotic missions to planets, asteroids, and moons in the solar, and as a real-time system to alert crew to an enhanced radiation environment during orbital mission or space exploration.

The MIDN instrument for MidSTAR-1 consists of three sensor modules distributed around the spacecraft and a centralized electronics module that interfaces with the spacecraft power and communication systems, as illustrated in Figure 1(b). The external sensor module will be nearest the outside of the spacecraft but covered with thermal insulation to assure a nominal operating temperature. One internal sensor module will be fixed to the bottom of the spacecraft's first shelf. The other internal sensor module is centrally located on the bottom of the third shelf and surrounded by a polyethylene absorber to simulate shielding provided by human tissue and organs that enhance the contributions from secondary charged particles and neutrons. The mass breakdown for the instrument independent of the absorber is: 3 sensors modules, $<0.5 \mathrm{~kg}$ each; electronics module, $<1 \mathrm{~kg}$; and fasteners and cabling, $0.25 \mathrm{~kg}$; for a total of $<2.75 \mathrm{~kg}$, leaving a margin of $>0.25 \mathrm{~kg}$ for a total mass of $<3.0 \mathrm{~kg}$. The polyethylene absorber has an additional mass of approximately $6 \mathrm{~kg}$. Each sensor module will be connected to the electronics module located on the topside of the second shelf of the spacecraft, see Figure 1(b). 


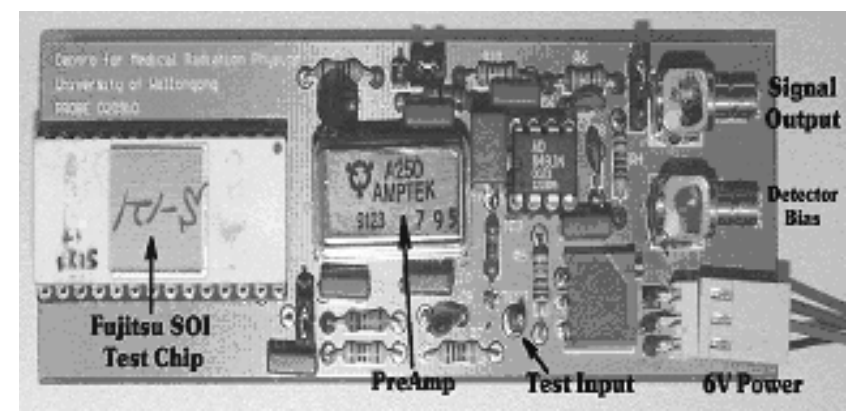

Figure 2 Microdosimeter sensor module

The electronics module can be partitioned into six functions: power conditioning, filtering of the sensor outputs, power management, analog-to-digital conversion, signal processing and storage, and command and telemetry. The signal flow from each sensor module is transmitted through a separate path and is received in parallel at the logic device. This allows all signals to be simultaneously processed, the observations stored, and the observations eventually transmitted to the spacecraft command and data handling $(\mathrm{C} \& D H)$ system.

The array consists of 120 by 40 diodes connected in parallel for a total of 4800 with each diode junction having an area of 10 by 10 square microns with the area of each diode being 30 by 30 square microns. The total surface of the 4800 cell array is $0.043 \mathrm{~cm}^{2}$. The sensitivity volume of each cell is 1.7 by 30 by 30 cubic microns. The detector converts charge pulses by a ratio of approximately $1 \mathrm{MeV}$ to $44 \mathrm{mV}$ which is amplified by the AMPTEK A250 preamplifier. The output is relatively insensitive to bias voltage, so the available $6 \mathrm{~V}$ will be utilized ${ }^{15}$.

The signal flow of the instrument is illustrated in Figure 3 that illustrates the 3 sensor modules and the electronics module. Illustrated in the electronics module are the filter function consisting of differentiator and integrator, power management controlled by the comparator, analog to digital convertors, the central logic device, the memory storage device, and the telemetry input and output.

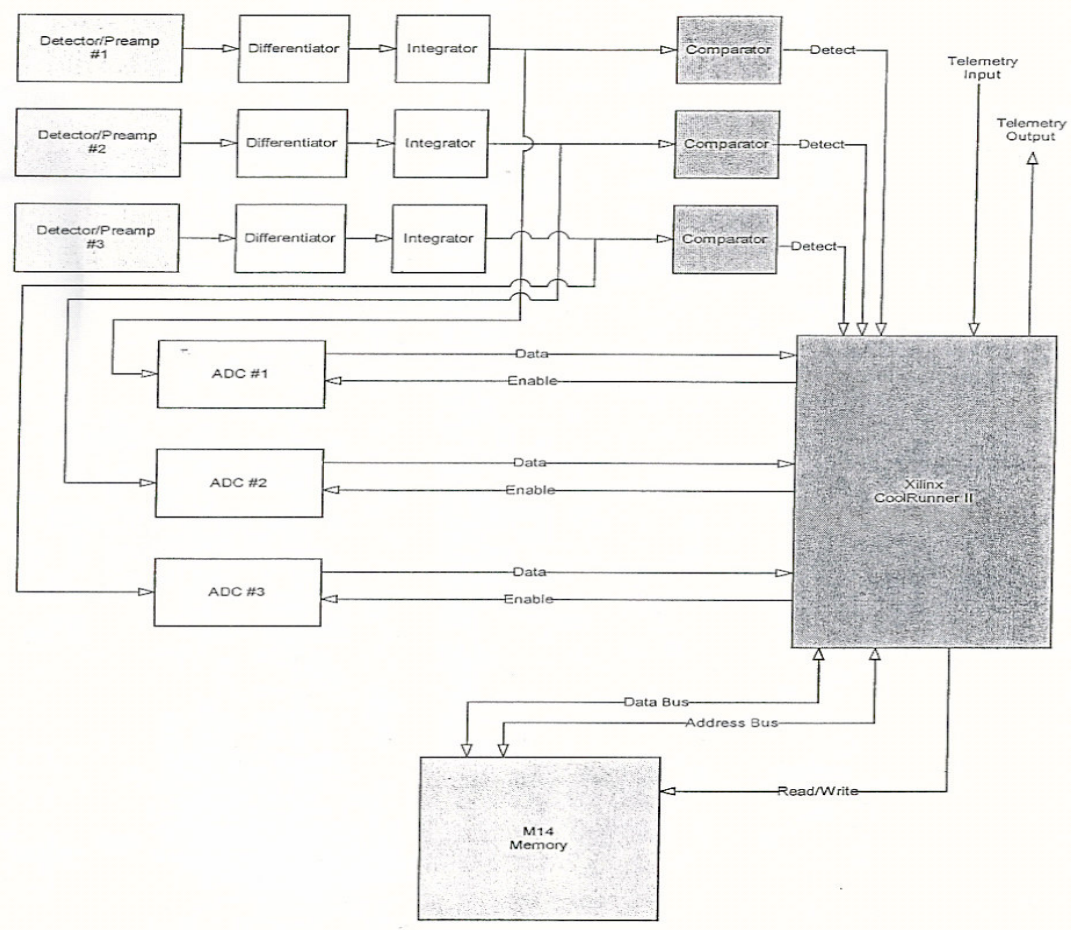

Figure 3 Instrument signal flow 
The electronic schematic of the instrument is given in Figure 4.

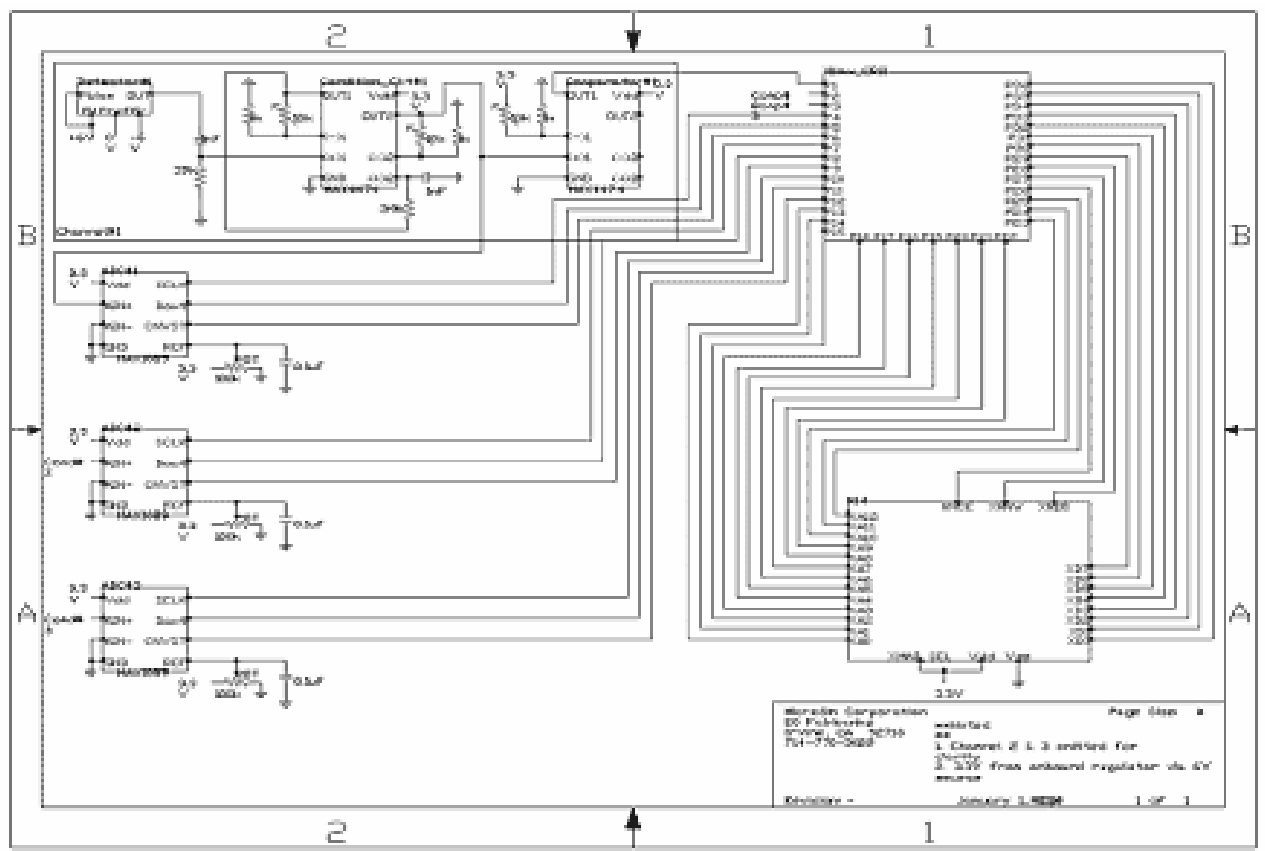

Figure 4 Electronic schematic

Signal conditioning consisting of high and low pass filtering (differentiator and integrator) is accomplished by utilizing the Maxim 4474 micropower operational amplifier and improves signal to noise and eliminates spurious counts. Logic processing is carried out in the Xilinx complex programmable logic device (CPLD) selected for its low power requirements. Power management carried out by the comparators is the key to developing a low-power system. When the voltage from the conditioning element is lower than threshold, the comparator notifies the CPLD which then withdraws power from the analog to digital converters (ADCs), signal processor, and the RS-422 communication chip creating a low power "sleep mode." The instrument remains in the alert "sleep mode" until the comparator receives a signal that is greater than a threshold voltage, at which point it activates the instrument. The Maxim MAX1089 10-bit ADC is used for the digital conversion. The outputs of the three ADCs go to the processor that consists of the CPLD and a proprietary memory chip, the M-14 manufactured by Medtronics Inc. The system was originally designed to operate with a CoolRunner-II 256 but will be upgraded to a 384 in order to support all three sensors. The M-14 memory is configured to require a few microwatts of power unless it is being accessed. Currently, a Digilent XC2-XL board with a RS-232 serial interface is being used to link with the spacecraft's C\&DH system. Upon completion of the initial testing regimen, the communications will be updated to RS-422 format.

A time constant for both integration and differentiation of 38 microseconds was determined to be optimal by preliminary tests ${ }^{15}$. This will eliminate high and low frequency noise picked up by the detector which is dominated by the $60 \mathrm{~Hz}$ due to the local environment. The spacecraft is anticipated to also have a somewhat noisy environment to be determined during spacecraft integration. This time constant selected is also short enough that multiple detections should not be misinterpreted as one observation. Figure 5 illustrates the filter element and the input square wave (top of Figure 5b) and output signal (bottom of Figure 5b) that represents the impulse response. 


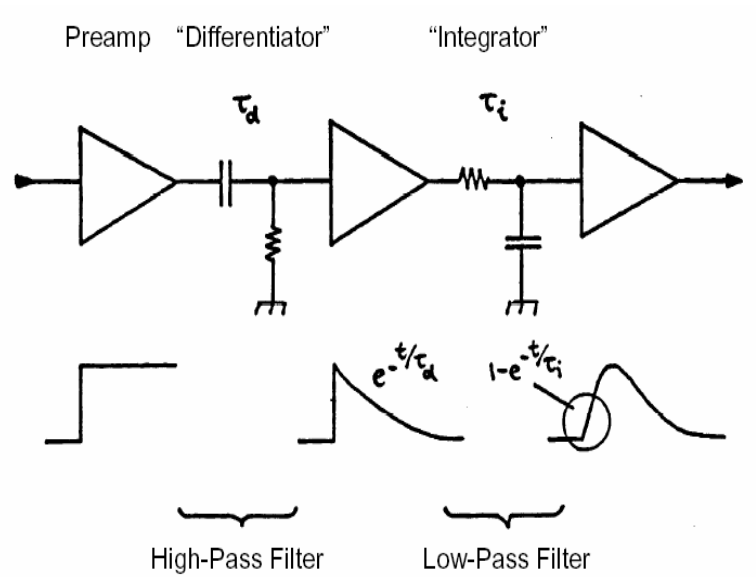

(a) Filter network

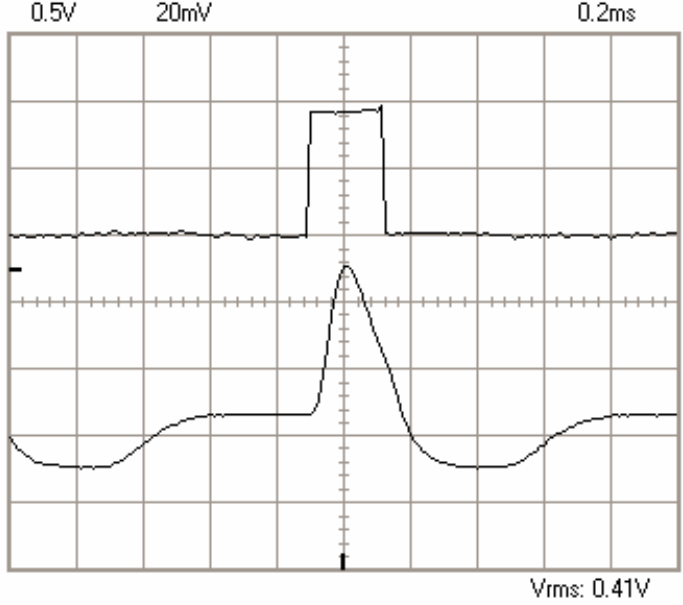

(b) Filter results

Figure 5 Filter network and results

The maximum power requirements were originally estimated as following: microdosimeter sensor module, $14 \mathrm{~mW}$; filters, $47 \mathrm{~mW}$; ADC, 1mW; XC2-XL board, $600 \mathrm{~mW}$ including the memory. Thus, the instrument was estimated to require a maximum of about $786 \mathrm{~mW}$ for a nominal one watt. However our tests indicate that independent of the three sensors at $14 \mathrm{~mW}$ each, the instrument is measured to require $540 \mathrm{~mW}$ in the operational mode. With the estimate of $14 \mathrm{~mW}$ for each sensor it means that the total power required should be about $582 \mathrm{~mW}$, significantly less than the original estimate. This is the power required only when the comparators indicate that an observation is to be processed. Remarkably, the memory draws a few microwatt of power in the "sleep mode" and the XC2-XL draws on the order of ten microwatts of power in the "sleep mode." The instrument can be powered off, powered on, and the stored data transmitted under control of the spacecraft command and data handling system. In the "sleep mode," while waiting to make an observation, the instrument draws less than $200 \mathrm{~mW}$. When powered off, the instrument draws no power.

A hardware simulator has been developed and successfully integrated with the spacecraft C\&DH system. As illustrated in Figure 6, it consists of the CPLD and the communications port. The CPLD has been programmed to receive and acknowledge commands and supply a fixed set of observations as if they were accessed from the memory.

The engineering model of the MIDN instrument is shown in Figure 7. The microdosimeter sensor with preamplifier is in the upper left, below it is the filter element and comparator element still in discrete form, below this is the link to the spacecraft $\mathrm{C} \& \mathrm{DH}$ system, now simulated by a personal computer. In the middle is the Xilinx programmer interface. To its right is the memory and below it is the Xilinx CPLD. In the final design the system will consist of the sensor modules with all other electronics packaged in a single electronics module. 


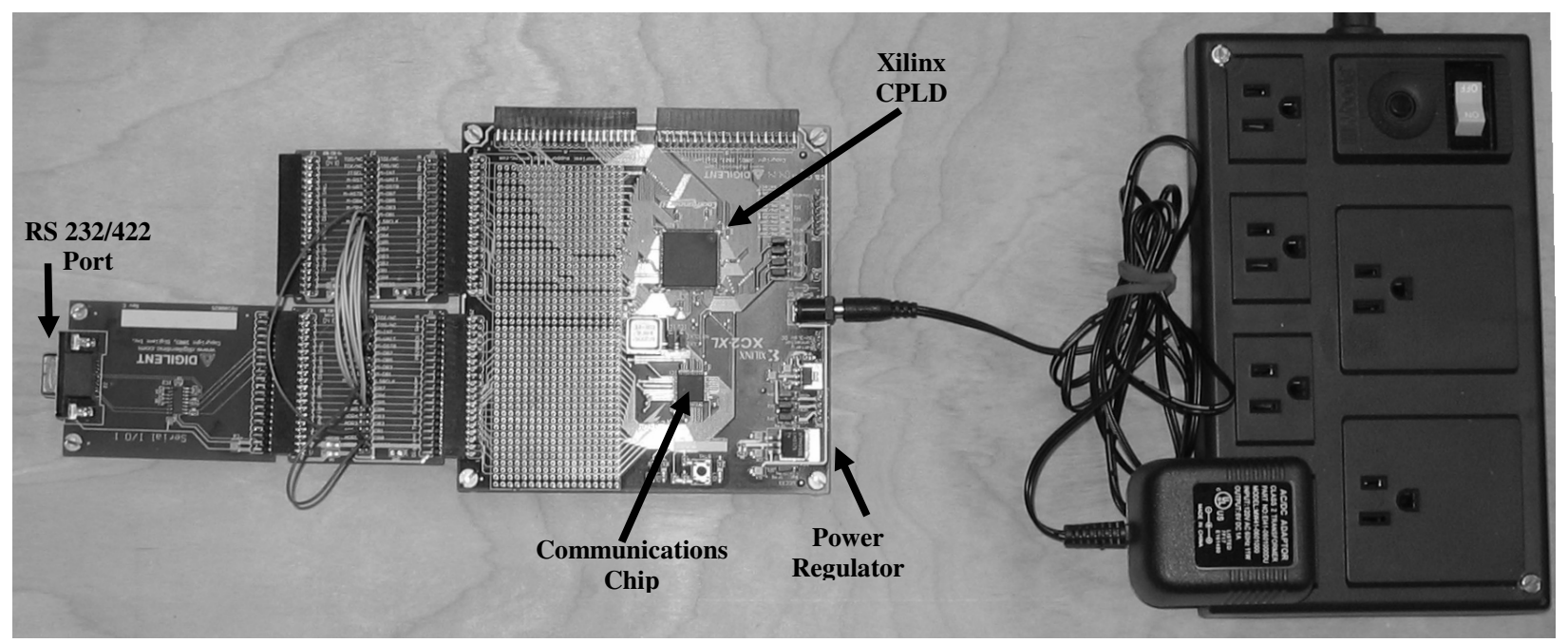

Figure 6 Instrument hardware simulator

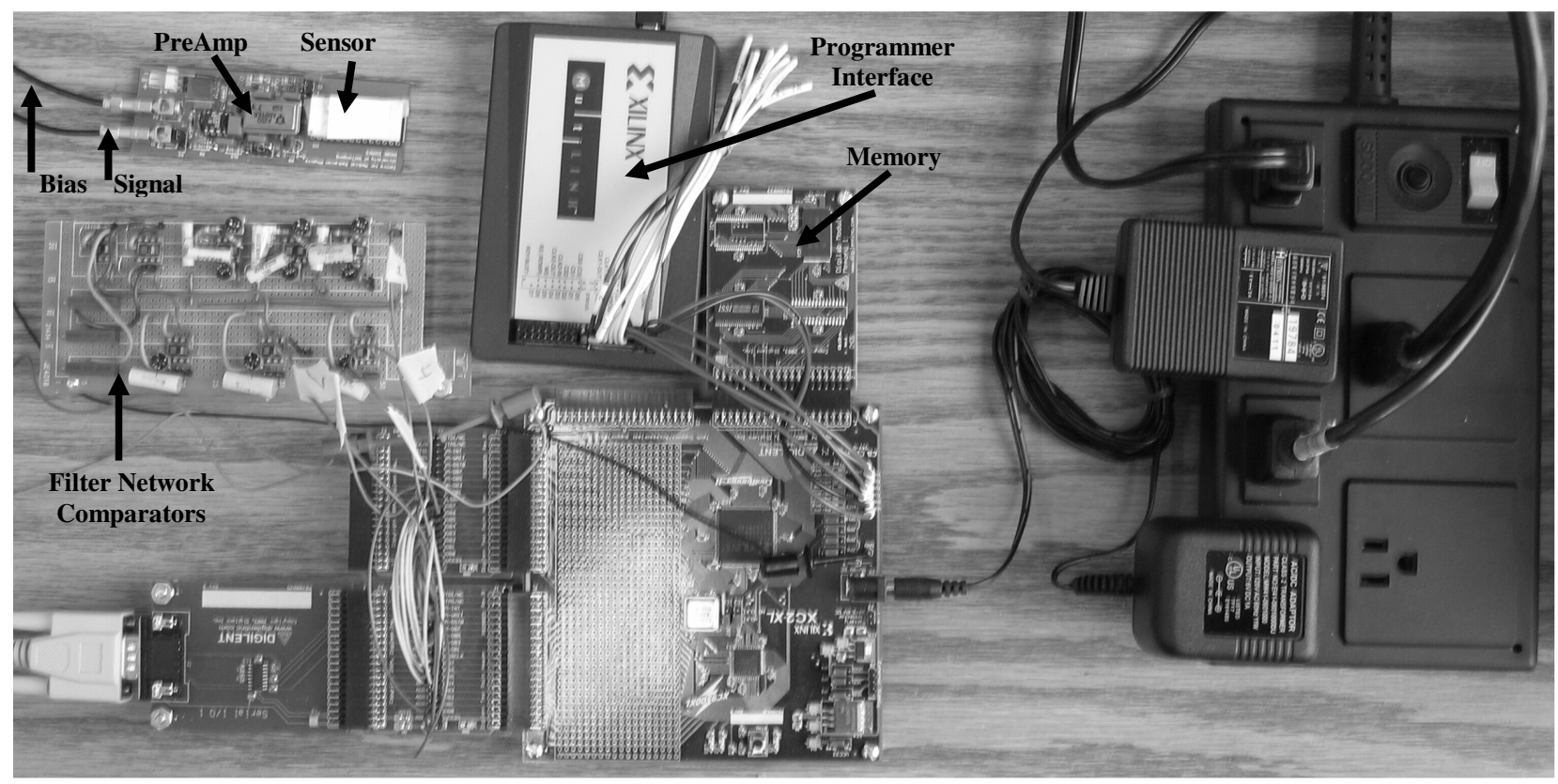

Figure 7 MIDN engineering model undergoing test

\section{Satellite Operations}

The stored data will consist of 3 sets of 1024 bins at 32 bits for a total of 12.288 Kbytes. The spacecraft computer will read the memory at a minimum frequency of once per day to provide an integrated assessment; however, it will be capable of reading at a rate of up to 1440 times per day (once per minute) during unique radiation conditions if directed by the spacecraft. Thus, time averages of the radiation environment will be obtained. Upon receiving the "read out" command, the stored data will be transmitted at 9600 bits per second. At this rate, each read out will take 10.24 seconds. The USNA spacecraft control station (USNA/SCS) will operate the spacecraft. Commands will be sent indirectly to the MIDN system via the spacecraft C\&DH system; and telemetry will be recorded and made available to the investigators. There will be a total of three commands sent to each sensor: "acquire data," "stop and reset," and "read out." Each collection sequence will begin with the command "stop and reset" to reset the circuit logic. "Acquire data" will then be used to zero the counters and begin data acquisition. The command "read out" 
can be given at any subsequent time to stop data acquisition and to send all data to the spacecraft computer to be stored there for transmission to the ground station. The C\&DH system will be able to repeatedly give the "read out" command to read a data set until the "acquire data" command is given, at which point the counters will be zeroed and the process will begin once more. The lifetime of the mission is expected to be at least one year.

\section{Project Status}

An engineering model of the instrument has been developed and integrated and is currently in the system integration and testing phase. The sensor modules and the various elements of the electronics module have been individually tested. Testing of the signal from the sensor output to the filtering element, comparator, and analog to digital conversion, comparator, and CPLD has been completed. Testing of the memory storage and readout under various scenarios is underway. In these tests, the radiation event is simulated using a $15 \mathrm{mV}$ pulse. When these preliminary tests are completed the instruments will be moved to the Radiation Laboratory to undergo final testing with radiation sources. The link to the MidSTAR-1 C\&DH system using the hardware simulator, described above, has passed initial tests.

\section{Initial Environmental Analysis}

An initial assessment of the ambient environment for the MidSTAR-1 spacecraft orbit was made using the SPace ENVironment Information System (SPENVIS) simulation ${ }^{16}$. This model includes trapped protons and electrons, solar ions, and cosmic ray ions. The models used for the trapped radiation are the AP-8 for protons and the AE- 8 for electrons. For solar protons, the one utilized was the JPL-91. An internal geomagnetic field was utilized without the external field because of the low altitude of the spacecraft. The estimates are based solely on the trapped radiation, as the model shows that essentially no solar particles or galactic cosmic rays (GCR) reach the low-altitude of the MidSTAR-1 orbit. The average undisturbed environmental trapped proton flux over a range of $0.1 \mathrm{MeV}$ to $400 \mathrm{MeV}$ is $124 \mathrm{~cm}^{-2} \mathrm{~s}^{-1}$ or 5.3 protons-s ${ }^{-1}$ for the array, or an upper bound of 30,000 protons per revolution, as all will not penetrate the spacecraft structure. The average undisturbed environmental trapped electron flux over a range of 0.1 $\mathrm{MeV}$ to $400 \mathrm{MeV}$ is $10^{6} \mathrm{~cm}^{-2} \mathrm{~s}^{-1}$ that for the array area gives 43,181 electrons- $\mathrm{s}^{-1}$ or an upper bound of 245,000 electrons per revolution, few of which will penetrate the spacecraft structure. If we assume a lower energy cutoff of $10 \mathrm{MeV}$ protons, then the average flux is about $108 \mathrm{~cm}^{-2} \mathrm{~s}^{-1}$ so the array area gives 4.6 protons-s $\mathrm{s}^{-1}$ or 26,000 protons per orbit. The effect of the structure will be considered in subsequent analyses.

\section{Summary}

A prototype of a small-mass, low-power, rugged microdosimetric system is being developed for space applications. All initial design goals appear to be achievable. The deployment of the proposed instrument as part of the MidSTAR-I mission will provide an opportunity to evaluate radiation fields for this mission and determine potential health risks under such conditions. This flight opportunity will provide the capability to evaluate a preliminary version of the microdosimetry system. It will also provide an opportunity to establish the capabilities and usefulness of the system and to compare the observations with the simulation of the ambient environment to assess the consistency between theory and observations. The project has successfully passed its conceptual design review and is in the construction and testing phase prior to a critical design review. The layout for the flight hardware has been completed and will be implemented in parallel with the critical design review. Delivery to the spacecraft for integration is set for 1 April 2005 with launch scheduled for September $1^{\text {st }}, 2006$.

\section{Acknowledgments}

This work is supported by the National Space Biomedical Research Institute through NASA NCC 9-58. Dr. Pisacane's chair is supported by the R. A. Heinlein Endowment Fund. 


\section{References}

1 NASA, "Bioastronautics Critical Path Roadmap (BCRP)," JSC 62577, NASA-Johnson Space Flight Center, Houston TX, 2 April 2004.

2 Dicello JF. HZE Cosmic Rays in Space: Is It Possible That They Are Not the Major Radiation Hazard? Radiat.Prot.Dosimetry 1992;253-8.

3 Space Studies Board. Radiation Hazards to Crews of Interplanetary Missions: Biological Issues and Research Strategies. National Research Council. 1996. Washington, D.C., National Academy Press.

Ref Type: Report.

4 National Council on Radiation Protection and Measurements. Radiation Protection Guidance for Activities in Low-Earth Orbit. National Council on Radiation Protection and Measurements. NCRP Report 132. 2000. Bethesda, MD, National Council on Radiation Protection and Measurements. Ref Type: Report.

5 F.A. Cucinotta, G.D. Badhwar, P. Saganti,, W. Schimmerling, J.W. Wilson, L.E. Peterson, and J. Dicello, Space Radiation Cancer Risk Projections for Exploration Missions: Uncertainty Reduction and Mitigation. NASA TP-2002-210777.

6 National Council on Radiation Protection and Measurements. Fluence-Based and Microdosimetric Event-Based Methods for Radiation Protection in Space. NCRP Report 137. 2001. Bethesda, MD, National Council on Radiation Protection and Measurements. Ref Type: Report.

7 Classification of NASA Payloads, NASA Management Instruction NMI 8010.1A, Appendix C.

8 D. Boden, "United States Naval Academy Small Satellite Program," presented at ASEE National Conf, 2000.

9 R. Bruninga, "PCsat, A Naval Academy Amateur Radio Satellite," 2004.

10 J. F. Dicello, "Practical Implications of Microdosimetry," pp. 35-44. Ion Beams in Tumor Therapy, U. Linz, ed. Chapman \& Hall, NY, 1995.

11 ICRP 60, 1991: 1990 Recommendations of the International Commission on Radiological Protection. ICRP Publication 60. Pergamon Press.

12 A. Rosenfeld, "Semiconductor microdosimetry in mixed radiation field: present and future," Radiation Protection Dosimetry, vol. 84, N1-4, pp. 385-388, 1999.

13 P. Bradley, Ph.D. Thesis "The Development of a Novel Silicon Microdosimeter for High LET Radiation Therapy," under the supervision of A.B. Rozenfeld in Department of Engineering Physics. NSW, Australia: University of Wollongong, 2000, pp. 333.

14 V. L. Pisacane, J. F. Ziegler, M. E. Nelson, M. Caylor, D. Flake, L. Heyen, E. ZYoungborg, A. B. Rosenfeld, F. A. Cucinotta, M. Zaider, and J. F. Dicello, MIDN, A Spacecraft Microdosimeter Mission, (accepted by Radiation Protection Dosimetry).

15 R. Callahan, "Microdosimetry Satellite Project," Research Report, United States Naval Academy 2003.

16 SPENVIS, "SPENVIS, Space Environment Information System," (http://www.spenvis.oma.be/spenvis/), 2004. 